\title{
MORBIDITY AND MORTALITY RATIO IN SURGICALLY TREATED FRAGILITY FRACTURES OF HIP IN ELDERLY POPULATION AT GOA MEDICAL COLLEGE HOSPITAL, GOA, INDIA (2011 - 2015)
}

\author{
Shivanand M. Bandekar1, Trivikram², Ashok Velip ${ }^{3}$ \\ ${ }^{1}$ HOD, Department of Orthopaedics, Goa Medical College. \\ ${ }^{2}$ Senior Resident, Department of Orthopaedics, Goa Medical College. \\ 3Junior Resident, Department of Orthopaedics, Goa Medical College.
}

\section{ABSTRACT}

\section{BACKGROUND}

Hip fractures are a major cause of morbidity and mortality in elderly population. ${ }^{1}$ Incidence of fragility fractures is increasing in Asia and in India.2,3 There is a significant variation in incidence of hip fractures in different regions of a country and among different socioeconomic status. 4,5

\section{MATERIALS AND METHODS}

The present study evaluates retrospectively the type of hip fractures, age, sex distribution, type of surgery, stay in hospital, side of injury, medical illness, associated injuries, cause of death, complications among 1181 patients admitted in Goa Medical College during the year 2011 - 2015.

\section{RESULTS}

As per the inclusion criteria, 966 patients were included in the study; $36.02 \%$ were males and $62.98 \%$ were females; mean age of $73.10 \%$ with $45.5 \%$ within 60 - 70 years of age. There were 496 left- and 470 right-sided cases; $58.48 \%$ were intertrochanteric fractures and $28.67 \%$ neck of femur, subtrochanteric $8.0 \%$ and basicervical $2.98 \%$; 561 patients underwent DHS fixation, 259 hemiarthroplasty, $40 \mathrm{PFN}$ and 44 others like DCS, THR, trochanteric buttress plate; $44.9 \%$ patients required preoperative transfusion, $20.5 \%$ postop. Duration of stay was average 11.65 days; $50.7 \%$ cases postop stay was less than 3 days. Uncontrolled DM and patients on anticoagulants were the main cause of delay in surgery in many patients. Only 138 (15.2\%) patients were without medical co-morbidities. There were 62 cases of death with 26 males and 36 females. Mean age of 76.5. Of the 62 death, 19 (30.6\%) occurred within 48 hours of admission; $65 \%$ patients had acute events that led to the death like ACS, CVA, RS failure, SCD and pulmonary embolism.

\section{CONCLUSION}

There is a need for treatment of hip fractures on urgent basis on admission, i.e. within 36 hours and need for orthogeriatrics in perioperative care. There is also a need for national level hip fracture management programme and hip fracture registry standardise and to monitor the quality of care.

\section{KEYWORDS}

Elderly Population, HIP Fractures, Osteoporosis, Perioperative Care.

HOW TO CITE THIS ARTICLE: Bandekar SM, Trivikram, Velip A. Morbidity and mortality ratio in surgically treated fragility fractures of hip in elderly population at Goa Medical College Hospital, Goa, India (2011 - 2015). J. Evolution Med. Dent. Sci. 2017;6(27):2270-2274, DOI: 10.14260/Jemds/2017/488

\begin{abstract}
BACKGROUND
Incidence of hip fractures is increasing in elderly population worldwide. ${ }^{1}$ There is a significant variation in incidence among different countries of the world and within a country. 4,5 India being a country with diverse population and regions, is bound to have a variable incidence and epidemiological profile of patients with hip fractures. There is lack of "Hip fracture registry" and a lack of uniform protocol for managing hip fracture patients. Majority of Hip fracture patients are referred to tertiary care Institutes for management as they have multiple co-morbidities and need
\end{abstract}

Financial or Other, Competing Interest: None.

Submission 20-02-2017, Peer Review 25-03-2017,

Acceptance 27-03-2017, Published 03-04-2017.

Corresponding Author:

Trivikram,

Room No. 246,

GARD Hostel Opposite Goa Medical College,

Bambolim, Goa- 403202.

E-mail: trivikrambht@gmail.com

DOI: $10.14260 /$ jemds $/ 2017 / 488$
ICU/HDU care. We evaluated retospectively all the patients admitted to Goa Medical College from period of 2011 - 2015 with hip fractures. The aim was to evaluate the epidemiological profile, treatment given, post-operative care, complications and compare it with that of International Institutes like the NHS U.K in order to standerdise the care and identify the shortcomings in treatment protocol followed by us.

\section{MATERIALS AND METHODS}

This is a retrospective observational study conducted in Goa Medical College Hospital, Goa. The study included patients admitted between Dec 2015 to Jan 2011 with fragility fractures of hip.

Patients with high velocity injuries and medicolegal cases were excluded from study. Patients less than 60 years of age and patients treated conservatively were excluded.

The medical records of the hospital were accessed for obtaining the data regarding the patients. The data collected included name, age, sex, duration of stay, diagnosis, associated injuries, associated medical illness, $\mathrm{Hb} / \mathrm{PCV}$, 
postop complications, transfusion requirements, postop stay, type of surgery done, postop and other significant events during the stay and outcomes such as death.

The data was tabulated and compared with published data and International Standards like the NHS.

\section{RESULTS}

A total of 1181 patients were admitted in Goa Medical College during period of 2011 - 2015 with fragility fractures of the hip. Out of 1181 patients, 966 were included in the study as per inclusion and exclusion criteria.

Out of 966 patients, 348 were males (36.02\%) and 618 were females $(63.90 \%)$.

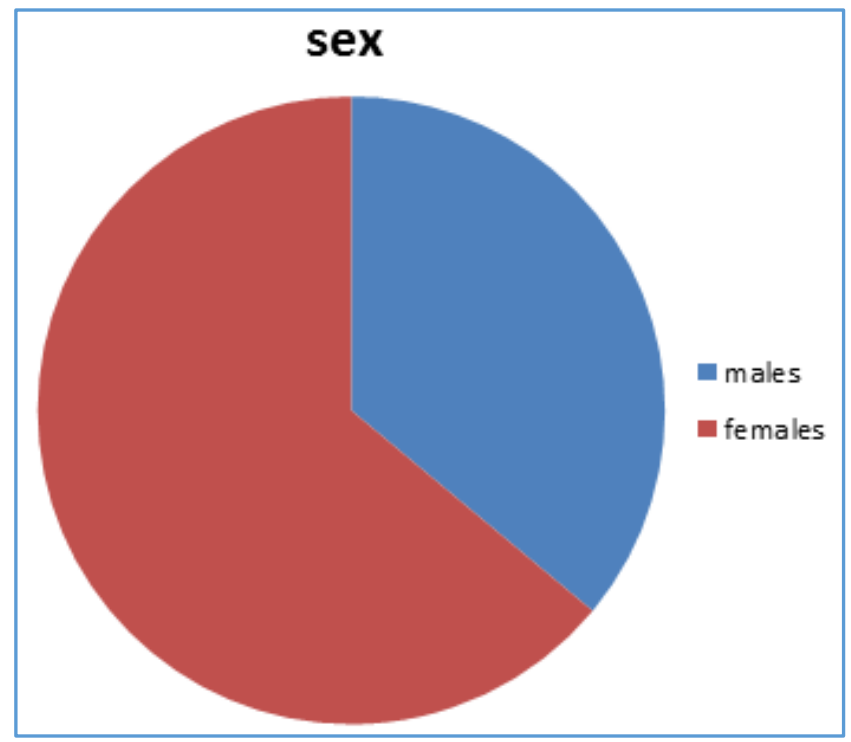

Majority of the patients were in between 60 - 80 years of age with $60-70$ age $=443(45.85 \%)$ patients and $70-80$ age $=362(37.47 \%)$ patients; 145 patients were in $81-90$ years age, that is (15.01\%). Only 17 (1.75\%) patients were in more than 91 years' age group. There was a wide range in distribution of patients from 60 - 108 years with mean age of 73.10 and an SD of 8.423.

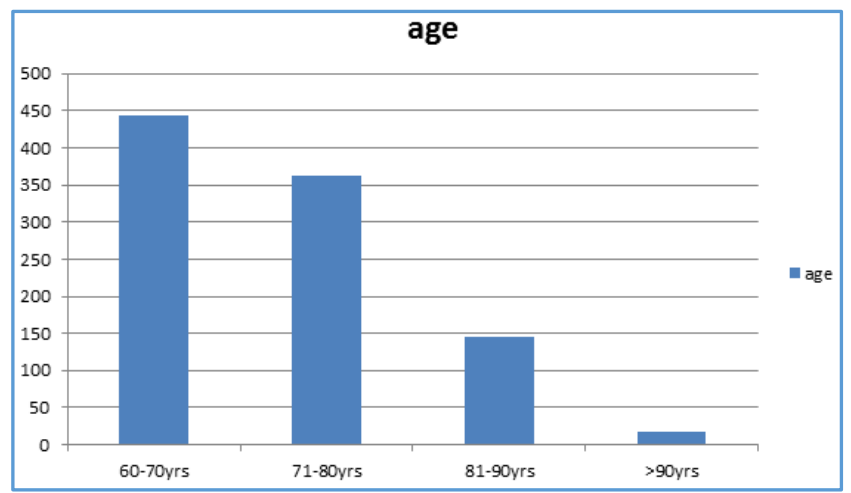

Out of 966 patients, major type was intertrochanteric fracture with 565 patients (58.48\%), second common type of fracture was neck of femur fracture 277 patients $(28.67 \%)$. Subtrochanteric fracture was the third most common with 78 patients (8.07\%); 27 patients were admitted with diagnosis of basicervical fracture consisting of $2.98 \%$.

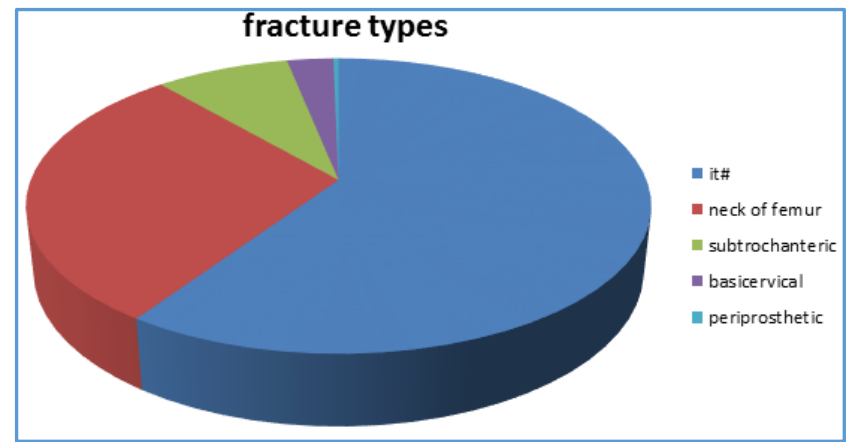

13 patients were admitted with postop infection (1.4\%); 3 patients were admitted with diagnosis of periprosthetic fracture $(0.33 \%)$; 1 patient with avascular necrosis of head of femur post fracture neck underwent THR.

1 patient with fracture neck of femur and psychiatric illness had dislocation twice and underwent girdlestone arthroplasty. The remaining patients are from the death statistics in each group; 25 patients with IT\#, 17 with neck of femur fracture and 20 patients with subtrochanteric fracture.

Patients were treated as follows Intertrochanteric Fracture - 540 Patients Operated

\begin{tabular}{|c|c|c|}
\hline DHS & 503 & $93.1 \%$ \\
\hline DCS & 3 & $0.55 \%$ \\
\hline PFN & 23 & $4.25 \%$ \\
\hline Trochanteric Buttress Plating & 1 & $0.18 \%$ \\
\hline Hemiarthroplasty & 9 & $1.66 \%$ \\
\hline Total Hip Arthroplasty & 1 & $0.18 \%$ \\
\hline
\end{tabular}

Out of 540 patients operated for intertrochanteric fracture $93.1 \%$ patients underwent dynamic hip screw fixation, proximal femoral nailing was next common type in $4.25 \%$ patients, $1.66 \%$ patients underwent hemiarthroplasty and dynamic condylar screw, trochanteric buttress plate and total hip arthroplasty was treatment options in less than $1 \%$ of total patients.

\section{Fracture Neck of Femur - 260 Patients Operated}

\begin{tabular}{|c|c|c|}
\hline Hemiarthroplasty & 246 & $94.61 \%$ \\
\hline Total Hip Arthroplasty & 2 & $0.76 \%$ \\
\hline Osteosynthesis & 2 & $0.76 \%$ \\
\hline DHS & 10 & $3.84 \%$ \\
\hline
\end{tabular}

$94.61 \%$ of patients with fracture neck of femur underwent hemiarthroplasty with uni/bipolar prosthesis; $3.84 \%$ patients underwent DHS; osteosynthesis and total hip was treatment option in 2 patients each.

\section{Subtrochanteric Fracture - 58 Patients Operated}

\begin{tabular}{|c|c|c|}
\hline DHS & 25 & $43.10 \%$ \\
\hline DCS & 12 & $20.68 \%$ \\
\hline PFN & 17 & $29.31 \%$ \\
\hline ILN & 2 & $3.44 \%$ \\
\hline TBP & 2 & $3.44 \%$ \\
\hline
\end{tabular}


Subtrochanteric fracture was treated with DHS in $43.10 \%$ patients, proximal femoral nailing was treatment option in $29.31 \%$ patients, $20.68 \%$ underwent DCS, interlocking and trochanteric buttress plating was treatment option in $3.44 \%$ of patients each.

\section{Basicervical Fracture - 25 Patients Operated}

\begin{tabular}{|c|c|c|}
\hline DHS & 21 & $84 \%$ \\
\hline Hemiarthroplasty & 4 & $16 \%$ \\
\hline
\end{tabular}

Basicervical fracture was treated with DHS in 84\% patients and hemiarthroplasty in $16 \%$ patients.

2 patients who were admitted as cervico-trochanteric fracture were treated with DHS. Of the 3 patients with periprosthetic fractures, 2 underwent ORIF and 1 THR.

14 patients who were admitted with postop infection, 7 underwent lavage and 7 implant removal.

1 patient with postop fracture neck of femur with AVN underwent THR.

2 cases of dislocated hemiarthroplasty underwent open reduction.

\section{Operative Time was Variable}

\begin{tabular}{|c|c|c|}
\hline Time in Min & No. of Patients & Percentage \\
\hline$<45$ mins. & 5 & $0.55 \%$ \\
\hline $46-60$ mins. & 244 & $26.99 \%$ \\
\hline $61-90$ mins. & 532 & $58.84 \%$ \\
\hline $91-120$ mins. & 88 & $9.73 \%$ \\
\hline $121-180$ mins. & 30 & $3.31 \%$ \\
\hline$>180$ mins. & 5 & $0.55 \%$ \\
\hline
\end{tabular}

532 (58.8\%) patients were operated within 61 - 90 mins.; $243(26.9 \%)$ patients have been operated within 60 mins. There were 88 patients in whom operative time was 1.5 - 2 hours and 30 patients with operative time up to 3 hours. And in the extreme were 5 patients with operative time of $<45$ minutes and 5 patients with operative time more than 3 hours.

$406(44.9 \%)$ required preoperative transfusion to bring the $\mathrm{Hb}$ to $10 \mathrm{~g}$. Postoperative transfusion was required in 186 patients $(20.57 \%)$.

Out of the operated patients, 496 were left side (51.3\%) and 470 were right (48.6\%).

Average stay in the hospital was 11.65 days with $50.7 \%$ patients staying less than 3 days postop. Standard deviation of 6.06. Most cases were delayed because of associated medical illness, patients on clopidogrel, time for arrangement of funds, etc. Most of the patients had multiple medical illnesses. With many patients having 2 or more medical illnesses, hypertension was the most common illness with 567 patients followed by diabetes mellitus 316 patients. Ischaemic heart disease was present in 75 patients and 60 patients had suffered CVA in past. There were 53 patients with COPD, 32 asthma and 26 alcoholic liver disease. Past history of malignancy was present in 14 patients and 12 were known case of CKD. Only 138 patients had no associated medical co-morbidities.

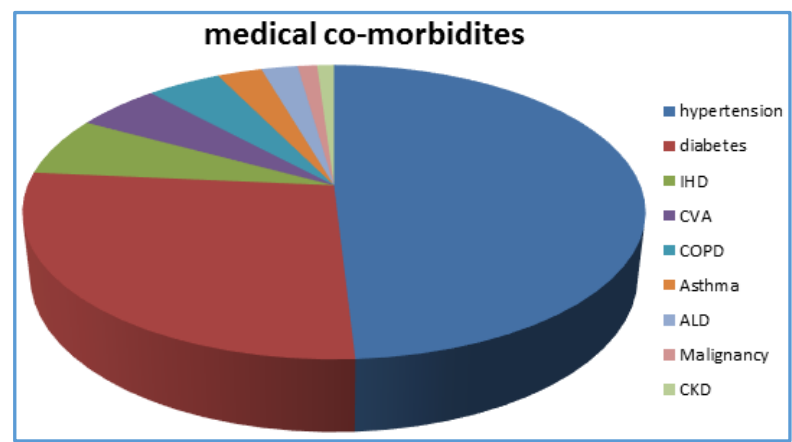

880 patients had no associated injuries; there were 5 patients with proximal humerus fracture, 4 on same side and 1 on opposite side; 10 cases of distal radius fracture 9 on same side and 1 on opposite side. Other injuries were 2 cases of spine fractures, 2 cases of both bones fracture, 1 case each of shaft femur, volar Barton's, head injury, elbow dislocation and orbital fracture.

Patients were monitored for postoperative complications intra and postop like acute coronary event, respiratory tract infections and respiratory failure, neurologic like CVA, renal complications like de-ranged RFT and hyponatraemia, DVT and pulmonary embolism, metabolic complications like uncontrolled DM postop, need for blood transfusion, revision and mortality.

\begin{tabular}{|c|c|c|}
\hline Complications & No. of Patients & Percentage \\
\hline CVS & 8 & $0.8 \%$ \\
\hline RS & 6 & $0.6 \%$ \\
\hline CNS & 2 & $0.2 \%$ \\
\hline Renal & 7 & $0.77 \%$ \\
\hline $\begin{array}{c}\text { DVT/Pulmonary } \\
\text { Embolism }\end{array}$ & 2 & $0.2 \%$ \\
\hline Blood Transfusion & 186 & $20.5 \%$ \\
\hline Revision Surgery & 3 & $0.33 \%$ \\
\hline
\end{tabular}

Of the 904 patients operated, most common complication encountered was need for postop blood transfusion in $20.5 \%$ patients, renal complications like de-ranged RFT and hyponatraemia in $0.77 \%$ patients, respiratory complication in $0.6 \%$, revision surgery in $0.33 \%$, CNS complication, DVT/Pulmonary embolism in $0.2 \%$ patients each.

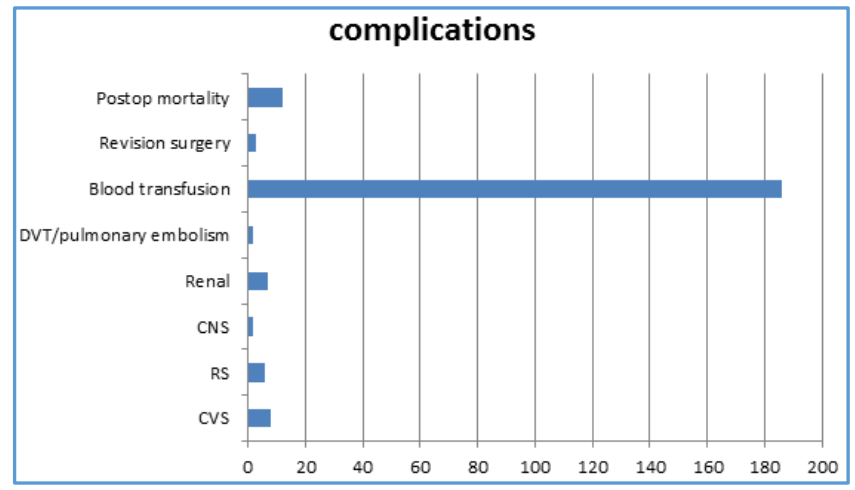

Remaining 678 patients postop period was uneventful (75\% patients).

On evaluation of death statistics in the Institution, following findings were obtained.

There were total of 67 cases of death in total of 1181 patients admitted and 62 were included as per inclusion criteria. 
26 patients were male and 36 females. There were 10 patients each in age group of 60 - 70 and 70 - 80 years among males; 5 patients in the age group of 81 - 90 years and 1 in the age group of 91 - 100 years. Among females, 55.55\% were in age group of $71-80$ and $33.33 \%$ patients in age group of 81 - 90. Remaining $11.11 \%$ patients were in age group of $60-$ 70 years. Overall, there were more female patients than male and overall age group was higher among females with majority more than 70 years.

\section{The Age Distribution was}

\begin{tabular}{|c|c|c|}
\hline Age Group & Males & Females \\
\hline $60-70$ & $10(38.4 \%)$ & $4(11.11 \%)$ \\
\hline $71-80$ & $10(38.4 \%)$ & $20(55.55 \%)$ \\
\hline $81-90$ & $5(19.23 \%)$ & $12(33.33 \%)$ \\
\hline $91-100$ & $1(3.84 \%)$ & 0 \\
\hline Total & $\mathbf{2 6}$ & $\mathbf{3 6}$ \\
\hline
\end{tabular}

Mean age was 76.51 with standard deviation of 8.29

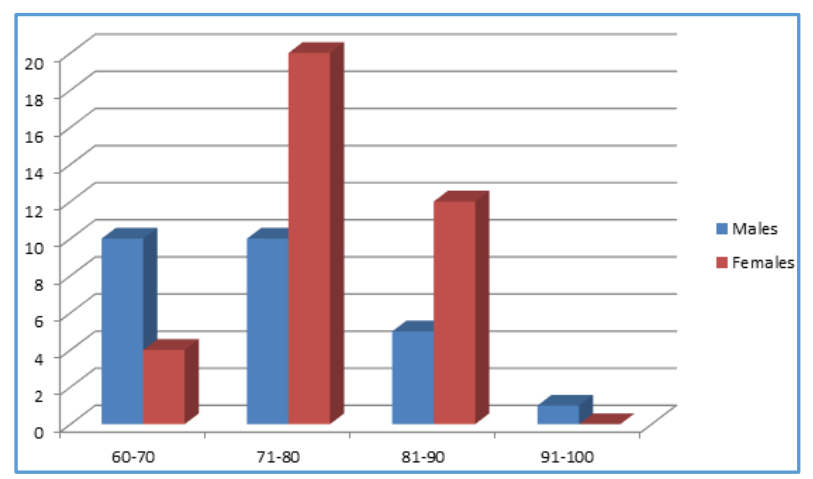

Mean stay in the hospital was 10.625 and standard deviation of 11.80 .

There were 25 patients with intertrochanteric fracture, 17 patients with neck of femur fracture and 20 patients with subtrochanteric fracture.

Of the 62 deaths, $19(30.6 \%)$ occurred within 48 hours of admission; $65 \%$ patients had acute events that led to the death like ACS, CVA, RS failure, SCD, pulmonary embolism.

There were 12 patients with cause of death as postop sepsis, 15 with sudden cardiac death, 3 with aspiration pneumonia, 8 with respiratory failure, 9 acute coronary event, 7 pulmonary embolism, 3 CVA, 2 metabolic encephalopathy, 2 UGI bleed and 1 CKD.

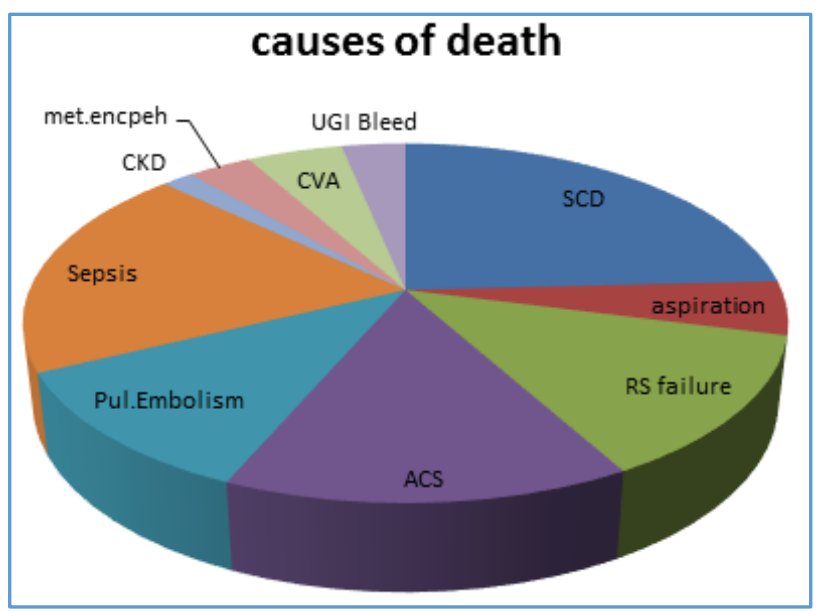

All the patients had multiple medical co-morbidities in addition to fracture with DM and HTN being most common.

\section{DISCUSSION}

Average age in our study population was 72.86 , this indicates that fragility fractures in the hip are important geriatric health problem. As many as $75 \%$ of geriatric population of India live in rural areas and elderly more than 70 years suffer from one or more chronic health problems. Joint pain and stiffness is the second most common morbidity after visual impairment as per ICMR Research. This makes elderly more prone for falls and fractures. ${ }^{6}$

The incidence of hip fractures among elderly is increasing worldwide. ${ }^{1}$ The incidence in U.K is $70-75,000$ cases with an annual cost of 2 billion pounds. 7,8

Incidence of fragility fractures is more common among females in our study and the average age among females was 73.56, which is in accordance to incidence worldwide. ${ }^{9,10}$

The average stay in our Institution was 11.76 days and average postop stay was 3.9 days. The cause of preoperative delay was mainly medical problems like DM, HTN and patients on clopidogrel which is as per Daniel Daniachi et al study in Brazil and Bentler et al in U.S.11,12,13

The delay in surgery was responsible for many complications like UTI, bedsores, LRTI, DVT. Hence, hip fractures in elderly need to be treated on urgent basis in hospital that is within 36 hours as per NICE guidelines. Hip fracture programme was implemented by Deepak Jain et al study which showed goal of early surgery, mobilisation and discharge from hospital with decreased mortality is achievable in Indian setting. ${ }^{14}$

$44.9 \%$ patients received preoperative transfusions and $20.57 \%$ patients received postoperative, this was as per the liberal transfusion protocol followed in our Institution wherein preoperatively $\mathrm{Hb}$ was raised to 10 and postoperatively patient received transfusion if $\mathrm{Hb}$ was less than 8 or severe pallor, major bleeding intraop. As we know, allogeneic blood transfusion is associated with significant cost and risks there is need to follow restricted transfusion protocol, wherein patient receives transfusion only if $\mathrm{Hb}$ is less than 7 which is safe and effective as per Cochrane review.15,16,17

Though for approximately $60 \%$ patients the postop stay was uneventful, remaining $40 \%$ had one or the other complications like hyponatraemia, altered sensorium, need for transfusion, pneumonia, etc. This stress the need for HDU care for patients with 1 organ compromise and ICU care for patients with 2 or more organ compromise. This is reflected in the mortality statistics, wherein $65 \%$ patients died of acute events like ACS, respiratory failure, pulmonary embolism, SCD. The mortality rate of $6.9 \%$ is as per various other studies by Sakaki et al, Daniel Dinachi et al and 6 months mortality as per Deepak Jain et al. But the mortality rate in US is $2 \%$ as per Bentler et al study. In our study, the risk of death increased with surgical delay of more than 48 hours which is as per numerous meta-analysis and systematic reviews in literature, which favour surgery within 48 hours as it leads to reduced 30 days mortality and 1 year mortality. ${ }^{18}$

\section{The Limitation of our Study are}

- The lack of long followup, which would help in knowing the functional outcome and mortality rates. 
- Representative population of the state is 1600000 , of which the study group is app 1000 which may appear insignificant as per country's population of 1.2 billion.

- Lastly, the limitations of manual record keeping followed at the Medical Records Department of the Institution may lead to some errors.

\section{CONCLUSION}

Hip fractures are primarily geriatric fractures and will increase in incidence as the aging population increases.

Patients are usually having multiple medical comorbidities and may have associated other fractures, hence the need for a Secondary or Tertiary Institute Care.

Hip fracture management needs team approach of "Orthogeriatrics" or Orthopaedician + physician + anaesthetist + physiotherapist team.

Hip fractures need to be treated on urgent basis, i.e. within 36 hours of admission to avoid the complications of bed rest and to reduce the mortality rate.

Need to develop detailed protocol to deliver better medical care in elderly.

\section{Suggested Protocol for Orthogeriatrics}

Step 1- Admission of elderly patients in orthogeriatrics ward and team management of the patients.

Step 2- Consider hip fracture as an emergency surgical case and rapid correction of de-ranged parameters.

Step 3- Stable fixation of the fracture to help in early mobilisation.

Step 4- Rapid transfer to dedicated HDU after surgery for < $48 \mathrm{hrs}$. post-surgery.

Step 5- Early mobilisation in the form of bed side sitting/chair sitting within $24 \mathrm{hrs}$. of surgery and walking with support in stable patients with help of physiotherapist.

\section{REFERENCES}

[1] Stevens JA, Rudd RA. The impact of decreasing U.S. hip fracture rates on future hip fracture estimates. Osteoporos Int 2013;24(10):2725-8.

[2] Macaulay W, Pagnotto MR, Iorio R, et al. Displaced femoral neck fractures in the elderly: hemiarthroplasty versus total hip arthroplasty. J Am Acad Orthop Surg 2006;14(5):287-93.

[3] Zi-Sheng A, You-Shui G, Zhi-Zhen J, et al. Hemiarthroplasty vs. primary total hip arthroplasty for displaced fractures of the femoral neck in the elderly: a meta-analysis. J Arthroplasty 2012;27(4):583-90.

[4] Falch JA, Kaastad TS, Bohler G, et al. Secular increase and geographical differences in hip fracture incidence in Norway. Bone 1993;14(4):643-5.
[5] Kaastad TS, Meyer HE, Falch JA. Incidence of hip fracture in Oslo, Norway: differences within the city. Bone 1998;22(2):175-8.

[6] Purty AJ, Bazroy J, Kar M, et al. Morbidity pattern among the elderly population in the rural area of Tamil Nadu, India. Turk J Med Sci 2006;36:45-50.

[7] www.nice.org.uk/guidance/CG124.

[8] Farnworth MG, Kenny P, Shiell A. The costs and effects of early discharge in the management of fractured hip. Age Ageing 1994;23(3):190-4.

[9] Hungria Neto JS, Dias CR, Bula de Almeidaet JD. Epidemiological characteristics and causes of proximal femoral fractures among the elderly. Rev Bras Ortop 2011;46(6):660-7.

[10] Ramalho AC, Lazaretti-Castro M, Hauache 0, et al. Osteoporotic fractures of proximal femur: clinical and epidemiological features in a population of the city of São Paulo. São Paulo Med J 2001;119(2):48-53.

[11] Mesquita GV, Lima M, Santos AMR, et al. Morbimortality in the elderly due to proximal fracture of the femur. Texto Contexto Enferm 2009;18(1):6773.

[12] Astur DC, Arliani GG, Balbachevsky D, et al. Fratura da extremidade proximal do fêmur tratadas no hospital São Paulo/Unifesp: estudo epidemiológico. Rev Bras Med 2013;68(4):11-5.

[13] Bentler SE, Liu L, Obrizan M, et al. The aftermath of hip fracture: discharge placement, functional status change, and mortality. Am J Epidemiol 2009;170(10):1290-9.

[14] Jain D, Sidhu GS, Selhi HS, et al. Early results of a geriatric hip fracture program in India for femoral neck fracture. Geriatr Orthop Surg Rehabil 2015;6(1):42-6.

[15] Carson JL, Hill S, Carless P, et al. Transfusion triggers: a systematic review of the literature. Transfus Med Rev 2002;16(3):187-99.

[16] Khan AM, Mushtaq N, Giannakas K, et al. Cross-match protocols for femoral neck fractures--finding one that can work. Ann R Coll Surg Engl 2004;86(1):11-4.

[17] Carless PA, Henry DA, Carson JL, et al. Transfusion thresholds and other strategies for guiding allogeneic red blood cell transfusion. Cochrane Database Syst Rev 2010;6(10):CD002042.

[18] Moja L, Piatti A, Pecoraro V, et al. Timing matters in hip fracture surgery: patients operated within 48 hours have better outcomes. A meta-analysis and meta-regression of over 190,000 patients. PLoS One 2012;7(10):e46175. 